\title{
Randomised controlled trial of azathioprine withdrawal in ulcerative colitis
}

\author{
A B Hawthorne, R F A Logan, C J Hawkey, P N Foster, A T R Axon, E T Swarbrick, B B Scott, \\ J E Lennard-Jones
}

\begin{abstract}
Objective-To determine whether azathioprine can prevent relapse in ulcerative colitis.

Design-One year placebo controlled double blind trial of withdrawal or continuation of azathioprine.

Setting-Outpatient clinics of five hospitals.

Subjects -79 patients with ulcerative colitis who had been taking azathioprine for six months or more. Patients in full remission for two months or more (67), and patients with chronic low grade or corticosteroid dependent disease (12) were randomised separately. 33 patients in remission received azathioprine and 34 placebo; five patients with chronic stable disease received azathioprine and seven placebo.
\end{abstract}

Main outcome measure-Rate of relapse. Relapse was defined as worsening of symptoms or sigmoidoscopic appearance.

Results-For the remission group the one year rate of relapse was $36 \%(12 / 33)$ for patients continuing azathioprine and $59 \%(20 / 34)$ for those taking placebo (hazard rate ratio $0.5,95 \%$ confidence interval 0.25 to $1 \cdot 0$ ). For the subgroup of 54 patients in long term remission (greater than six months before entry to trial) benefit was still evident, with a $31 \%(8 / 26)$ rate of relapse with azathioprine and $61 \%$ $(17 / 28)$ with placebo $(p<0 \cdot 01)$. For the small group of patients with chronic stable colitis (six were corticosteroid dependent and six had low grade symptoms) no benefit was found from continued azathioprine therapy. Adverse events were minimal.

Conclusions-Azathioprine maintenance treatment in ulcerative colitis is beneficial for at least two years if patients have achieved remission while taking the drug. Demonstration of the relapse preventing properties of azathioprine has implications for a large number of patients with troublesome ulcerative colitis, who may benefit from treatment with azathioprine.

\section{Introduction}

Since the first reports of the use of azathioprine and mercaptopurine in ulcerative colitis, ${ }^{1}$ there have been several uncontrolled studies reporting benefit but few placebo controlled trials. Two trials have shown evidence of a significant corticosteroid sparing effect in chronic active ulcerative colitis. ${ }^{23}$ However, in acute relapse Jewell and Truelove found no significant benefit from adding azathioprine to a standard regimen of corticosteroids. ${ }^{4}$ Some patients with chronic active or frequency relapsing disease are treated with azathioprine, and many of these achieve complete remission and can stop taking corticosteroids. ${ }^{5}$ The further management of these patients is unclear. In Jewell and Truelove's study the benefit of azathioprine maintenance treatment was equivocal, ${ }^{4}$ and many doctors are reluctant to continue prescribing the drug in view of the potential side effects of azathioprine, particularly bone marrow suppression. We designed a study to investigate whether continuing azathioprine treatment in these patients is beneficial.

\section{Subjects and methods}

We conducted a one year double blind withdrawa study in which patients who were already established on azathioprine were randomised to either continue the drug at the same dose or switch to an identical placebo. Approval from ethics committees in the participating hospitals was obtained, and all patients gave written informed consent. To be eligible for the study patients had to have had ulcerative colitis diagnosed on the basis of rectal biopsy and barium enema or colonoscopy and to have been taking azathioprine for a minimum of six months. Patients were categorised as being in remission or having chronic stable disease. Remission was defined as absence of symptoms of active disease in patients not taking corticosteroids and with a sig moidoscopic appearance of grade 0 or 1 (as described by Baron et $a l^{6}: 0=$ normal mucosa; $1=$ granular or oedematous mucosa with loss of vascular pattern 2 =bleeding to light touch; $3=$ spontaneous bleeding ahead of the instrument). Chronic stable disease was defined as low grade symptoms or symptom control with low doses of corticosteroids ( $10 \mathrm{mg}$ prednisolone or less) with a sigmoidoscopic appearance of grade 0 or 1. Patients had to have been in remission or in a stable state with no change in dose of prednisolone if taking corticosteroids for a minimum of two months before entering the trial. For both groups the study end poin was relapse, defined as worsening symptoms recog nised by the patient as active disease (such as recta bleeding, loose motions, or bowel frequency) with a sigmoidoscopic appearance of grade 1 or above or grade 2 or 3 appearance at routine sigmoidoscopy regardless of symptoms.

Patients were seen at entry to the trial, after one and two months, and then two monthly through the trial or earlier in the event of relapse. At each visit blood was taken for full blood count, liver function tests, and determination of erythrocyte sedimentation rate and $\alpha 1$ acid glycoprotein concentration. If the platelet count fell below $120 \times 10^{9} / 1$ or total white cell count below $3 \times 10^{9} / 1$ the trial drug was stopped temporarily and reintroduced at a lower dose as indicated. Sigmoidoscopy and rectal biopsy were performed at entry and at two, six, and 12 months or at time of relapse. Patients kept a daily symptom diary, which was reviewed at clinic visits and included a record of tablet consumption. Compliance was monitored by a record of tablet consumption in the diary cards. Relapse was dated from the onset of worsening symptoms as recorded in the diaries.

Trial treatment was either the same dose of azathioprine as previously taken or an equivalent number of placebo tablets of identical appearance. Randomisation was performed in hospital pharmacies in blocks of four. Trial drugs or placebo were dispensed by the hospital pharmacies using separate randomisation schedules for the patients in remission and with

Correspondence to: Dr Logan. 
chronic stable disease. Treatment with drugs containing 5-aminosalicylic acid was continued during the trial at the dose taken before entry.

We analysed the data initially by the Kaplan-Meier survival method using the generalised Wilcoxon test and the program KMSURV (Kaplan-Meier product limit estimates of $S(t)$, Ludwig Institute for Cancer Research, release Nov 1988). A Cox's proportional hazards survival analysis was also done with the program EGRET. Our previous survey had shown a probability of relapse of about one third for patients in established remission who continued to take azathioprine. ${ }^{5}$ To show an increase in relapse from $35 \%$ to $70 \%$ in those who stop taking azathioprine with a power of 0.8 (two tailed $\alpha=0.05$ ) 70 patients would be required.

\section{Results}

Patients were enrolled at five hospitals, with numbers from each hospital ranging from six to 39 . Sixty seven patients with ulcerative colitis in remission were enrolled; 34 were randomised to receive placebo and 33 to continue azathioprine. Twelve patients with chronic stable disease were randomised separately; five received placebo and seven continued azathioprine. The characteristics of patients were generally similar in all trial groups (table), although there was a greater number of men randomised to placebo in the remission group. Overall, half of the patients had total colitis, the median azathioprine dose was $100 \mathrm{mg}$, and 67 of the 79 patients were taking aminosalicylates.

\section{REMISSION GROUP}

One patient defaulted from follow up at eight months and another stopped trial treatment at 11 months because of a misunderstanding (both were taking azathioprine). These patients' data were censored at the time of protocol violation. Two patients in the remission group were found, after completing the trial, to have Crohn's disease rather than ulcerative colitis. These patients were included in the primary analysis, but a secondary analysis was performed which excluded them.

Twelve (36\%) of the 33 patients taking azathioprine relapsed compared with $20(59 \%)$ of the 34 patients taking placebo (fig; Wilcoxon $\chi^{2}$ statistic 4.26, $\mathrm{p}=\mathbf{0 . 0 3 9}$ ). Overall the relapse rate of those who continued taking azathioprine was half that of the patients who switched to placebo (hazard rate ratio 0.5 , $95 \%$ confidence interval 0.25 to $1 \cdot 0$ ). All of the patients who relapsed had symptoms and in none was relapse diagnosed on abnormal sigmoidoscopic appearance alone. A secondary analysis excluding the two patients shown subsequently to have Crohn's disease (both

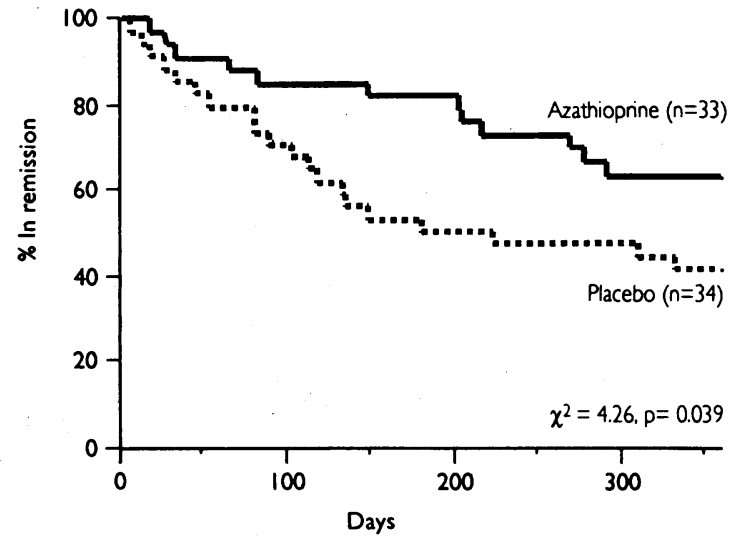

Kaplan-Meier survival plot of the rate of relapse over one year for 67 patients with ulcerative colitis in remission after taking azathioprine

were taking azathioprine and one relapsed) gave a relapse rate of $35 \%(11 / 31)$ for the azathioprine group compared with $59 \%$ for the placebo group $(p=0 \cdot 04)$. To investigate whether maintenance treatment was effective in patients with more prolonged remission or was restricted to those patients with a recent relapse a subgroup of 54 patients was identified who had been in remission for more than six months at entry to the trial. The one year relapse rate was $31 \%(8 / 26)$ for the patients taking azathioprine, and $61 \%(17 / 28)$ for those taking placebo $(\mathrm{p}=0.01)$.

In view of the modest trial size and the sex imbalance in the two treatment groups (table) the data were also analysed with Cox's proportional hazards model. The effects of sex, age, duration of remission before trial entry, and treatment were analysed independently and in combination. The relapse rate for women was similar to that for men (hazard rate ratio $1.03, p=0.93$. There was a highly significant fall in relapse rate with increasing age (hazard rate ratio for one year older $0.95,95 \%$ confidence interval 0.93 to $0.98 ; \mathrm{p}<0.001$ ). Longer duration of remission before trial entry was inversely related to relapse rate (hazard rate ratio 0.97 , 0.93 to $1 \cdot 01 ; p=0 \cdot 10$ ). In the model containing all four variables continued azathioprine treatment remained beneficial (hazard rate ratio $0.43,0.20$ to 0.93 ; $\mathrm{p}=0.03$ ); the strong association between relapse rate and age was unchanged.

\section{CHRONIC STABLE DISEASE GROUP}

In the chronic stable group one patient stopped taking sulphasalazine ( $4 \mathrm{~g}$ daily) at entry to the trial and subsequently stopped trial treatment because of ongoing chronic active symptoms (he was taking azathioprine) and changed to unblinded azathioprine at eight months. Two other patients in the chronic active

Characteristics of patients with ulcerative colitis in remission or with chronic stable disease randomised to receive placebo or azathioprine

\begin{tabular}{|c|c|c|c|c|}
\hline & \multicolumn{2}{|c|}{ Remission } & \multicolumn{2}{|c|}{ Chronic stable disease } \\
\hline & $\begin{array}{l}\text { Placebo } \\
(\mathrm{n}=34)\end{array}$ & $\begin{array}{l}\text { Azathioprine } \\
(\mathbf{n}=33)\end{array}$ & $\begin{array}{c}\text { Placebo } \\
(n=5)\end{array}$ & $\begin{array}{l}\text { Azathioprine } \\
(\mathbf{n}=7)\end{array}$ \\
\hline Mean (range) age (years) & $44(19-82)$ & $44(23-73)$ & $37(24-57)$ & $56(31-75)$ \\
\hline $\operatorname{Sex}(\mathbf{M} / \mathbf{F})$ & $22 / 12$ & $12 / 21$ & $4 / 1$ & $3 / 4$ \\
\hline \multicolumn{5}{|l|}{ Colitis extent: } \\
\hline Total & 19 & 18 & & 1 \\
\hline Left sided & 8 & 5 & 1 & 1 \\
\hline Sigmoid & 7 & 8 & 2 & 4 \\
\hline Proctitis & & 2 & 2 & 1 \\
\hline Mean (range) azathioprine dose (mg) & $100(50-150)$ & $100(50-200)$ & $150(100-150)$ & $100(50-150)$ \\
\hline \multicolumn{5}{|l|}{ Sulphasalazine: } \\
\hline Mean (range) dose (g) & $2(1-4)$ & $2(1-4)$ & $2(2-3)$ & $2(2-4)$ \\
\hline No of patients & $=22$ & 17 & 4 & \\
\hline \multicolumn{5}{|l|}{ Mesalazine: } \\
\hline Mean (range) dose (g) & $1 \cdot 2(1 \cdot 2-2 \cdot 4)$ & $1 \cdot 2(0 \cdot 8-3 \cdot 2)$ & & $1 \cdot 2(0 \cdot 8-1 \cdot 6)$ \\
\hline No or patients & 13 & 15 & & \\
\hline No of patients not taking aminosalicylates & 4 & 8 & 1 & 0 \\
\hline Mean (range) duration of disease before trial (years) & $7(1-28)$ & $9(2-30)$ & $3(3-6)$ & $9(3-32)$ \\
\hline Mean (range) duration of azathioprine treatment before trial (months) & $21(7-93)$ & $19(7-96)$ & $20(16-21)$ & $26(14-37)$ \\
\hline Mean (range) duration of remission before entry (months) & $11(4-45)$ & $12(2-48)$ & & \\
\hline
\end{tabular}


group stopped trial treatment at four and 10 months because of disillusionment with the trial (both taking placebo). These patients' data were censored at the time of protocol violation.

Of the 12 patients in the chronic stable group, two were receiving corticosteroid enemas at entry, four were taking oral prednisolone $(2 \cdot 5-10 \mathrm{mg})$, and six had low grade symptoms but were not taking prednisolone. Five of the seven patients taking azathioprine relapsed, two at one month, two at two months, and one at five and a half months. Of the five patients taking placebo, two relapsed (at one and nine months). One relapse was diagnosed on the basis of a grade 3 sigmoidoscopic appearance at a routine examination without change in symptoms (the patient had chronic low grade rectal bleeding and had had a grade 1 mucosal appearance at entry to the trial). The remainder of relapses were diagnosed on the basis of worsening symptoms or increasing corticosteroid requirements.

The only side effect observed during the trial was transient bone marrow suppression in two patients taking azathioprine. In one of these patients a low white cell count $\left(2 \cdot 7 \times 10^{9} / 1\right)$ at four months responded to dose reduction from 100 to $50 \mathrm{mg}$, and the patient was subsequently stabilised at $75 \mathrm{mg}$. The other patient had a low platelet count $\left(73 \times 10^{9} / 1\right)$, which responded to temporary stopping of treatment $(150 \mathrm{mg})$ with later stabilisation at $50 \mathrm{mg}$ daily.

\section{Discussion}

Our study has shown that maintenance treatment with azathioprine is beneficial as withdrawal resulted in a doubling of the relapse rate in patients who had achieved complete remission. These patients represent a selected but substantial group who had poorly controlled colitis as well as a good response to (and tolerance of) azathioprine. The likely size of this group is not clear; in Nottingham at least 35 patients are currently taking azathioprine for ulcerative colitis. Our results also raise the possibility that a wider group of patients with ulcerative colitis might benefit from initial treatment with azathioprine.

Our study shows that patients in prolonged remission of six months or more still benefit from continuing maintenance treatment. The total duration of benefit is unclear. The duration of remission before entry to the trial was a median of 12 months, so maintenance treatment seems to be beneficial for a minimum of two years. Most patients in this study were taking sulphasalazine or mesalazine, and these drugs were continued during the trial. The benefit from azathioprine maintenance treatment in preventing relapse is thus additive to the effects of these drugs.

The group of patients who had not achieved complete remission and stopped taking corticosteroids after six months' treatment with azathioprine was small. Our trial was therefore unable to reliably determine whether azathioprine should be continued in these patients. Although there seemed to be no benefit from continuing azathioprine, the concept of remission in these patients is somewhat artificial, and an end point of relapse can be difficult to decide, often being based on a patient's perception of worsening symptoms. These patients may have been non-compliant with treatment but there was no evidence for this on the basis of diary record of tablet consumption or changes in lymphocyte counts. For whatever reason not all patients will respond to azathioprine, and if patients are not in remission within six months after starting taking the drug our data suggest there is little value in continuing it.
Azathioprine has not been widely used in ulcerative colitis because of the lack of clear evidence of its value and concern about its toxicity. Two controlled trials have shown a corticosteroid sparing effect in chronic active disease, ${ }^{23}$ but no significant benefit was seen over one month in patients with acute relapse. ${ }^{4}$ This was, perhaps, predictable in view of the slow onset of action of azathioprine,? and the high response rate to the concomitant corticosteroids. Although the continuation of this trial into a maintenance phase failed to detect an overall significant benefit, there was a strong trend in favour of azathioprine in a subgroup of patients admitted with a relapse of established disease, suggesting likely therapeutic benefit. ${ }^{4}$ The use of azathioprine and its metabolite 6-mercaptopurine is better established in Crohn's disease, with evidence of benefit in chronic active disease ${ }^{8-10}$ and in the prevention of relapse. This was seen both in patients who had just achieved remission on the drug,${ }^{11}$ and in patients in established remission in whom azathioprine was withdrawn according to a protocol similar to that used in our study. ${ }^{12}$ Azathioprine can cause serious adverse drug reactions ${ }^{5}$ such as nausea, bone marrow depression, or diarrhoea. ${ }^{13}$ However, these are either idiosyncratic and evident early in treatment or, in the case of bone marrow depression, can be avoided by regula monitoring of the blood count.

We conclude that patients with ulcerative colitis who have achieved remission with azathioprine benefit from continuing azathioprine in addition to aminosalicylic acid preparations for a minimum of two years. Although azathioprine is not widely used in ulcerative colitis, there is a growing awareness of its value in inflammatory bowel disease, as evidenced by an increasing number of recent reports of experience with the drug. ${ }^{1417}$ Our results give these observations a sound basis and suggest that azathioprine should be used more widely in patients with poorly controlled ulcerative colitis.

1 Bean RHD. The treatment of chronic ulcerative colitis with 6-mercapopurine. Med f Aust 1962;2:592-3.

2 Kirk AP, Lennard-Jones JE. Controlled trial of azathioprine in chronic ulcerative colitis. BMF 1982;284:1291-2.

3 Rosenberg JL, Wall AJ, Levin B, Binder HJ, Kirsner JB. A controlled trial of azathioprine in the management of chronic ulcerative colitis. Gastroentero$\log y$ 1975;69:96-9.

4 Jewell DP, Truelove SC. Azathioprine in ulcerative colitis: final report on controlled therapeutic trial. $B M \mathcal{F}$ 1974;iv:627-30.

Hawthorne AB, Logan RFA, Hawkey CJ. Azathioprine in resistant ulcerative colitis. Gastroenterology 1989;96:A20

6 Baron JH, Connell AM, Lennard-Jones JE. Variation between observers describing mucosal appearances in proctocolitis. $B M \mathcal{F} 1964 ; \mathrm{i}: 89-92$.

7 Hawthorne AB, Hawkey CJ. Immunosuppressive drugs in inflammatory bowel disease: a review of their mechanisms of efficacy and place in therapy. Drugs 1989;38:267-88.

8 Present DH, Korelitz BI, Wisch JL, Glass JL, Sachar DB, Pasternack BS. Treatment of Crohn's disease with 6-mercaptopurine. N Engl f $\mathrm{Med}$ 1980;302:981-7.

9 Rosenberg JL, Levin B, Wall AJ, Kirsner JB. A controlled trial of azathioprine in Crohn's disease. American fourmal of Digestive Diseases 1975;20:721-6.

10 Willoughby JMT, Thomas JM, Sudweeks DM. Azathioprine and levamisole in Crohn's disease: a double blind controlled trial of one year's treatmen with long follow up. Gut 1990;31:A1193.

1 Willoughby JMT, Beckett J, Kumar P, Dawson AM. Controlled trial of azathioprine in Crohn's disease. Lancet 1971;ii:944-6.

12 O'Donoghue DP, Dawson AM, Powell-Tuck J, Bown RL, Lennard-Jones JL. Double-blind withdrawal trial of azathioprine as maintenance treatment for Crohn's disease. Lancet 1978;ii:955-7.

13 Cox JA, Daneshmend TK, Hawkey CJ, Logan RFA, Walt RP. Devastating diarrhoea due to azathioprine-management difficulty in inflammatory bowel disease. Gut 1989:29:686-8.

14 O'Brien JJ, Bayless JA. Use of azathioprine or 6-mercaptopurine in the treatment of Crohn's disease. Gastroenterology 1991;101:39-47.

15 Lobo AJ, Foster PN, Burke DA, Johnston D, Axon ATR. The role of azathioprine in the management of ulcerative colitis. Dis Colon Rectum 1990;33:182-5.

16 Verhave $M$, Winter HS, Grand RJ. Azathioprine in the treatment of children with inflammatory bowel disease. Pediatrics 1990;117:809-14.

17 Adler DJ, Korelitz BI. The therapeutic efficacy of 6-mercaptopurine in refractory ulcerative colitis. Am F Gastraenterol 1990;85:717-22.

(Accepted 29 April 1992) 